\title{
Polyneuropathy in hypothyroidism: clinical, electrophysiological and morphological findings in four cases
}

\author{
RAFFAELLO NEMNI, * EDO BOTTACCHI, ${ }^{*}$ RAFFAELLA FAZIO,* \\ ANGELO MAMOLI, $\ddagger$ MASSIMO CORBO, ${ }^{*}$ MASSIMO CAMERLINGO $\ddagger$ \\ GIUSEPPE GALARDI,* LUCIANO ERENBOURG, $†$ NICHOLAS CANAL*
}

From the Departments of Neurology* and Surgical Anatomy, $\dagger$ University of Milan, Istituto Scientifico $S$. Raffaele, Milan and the Division of Neurology and Neurophysiopathology, $\ddagger$ Ospedale Regionale, Aosta, Italy

SUMMARY Clinical, neurophysiological and morphological studies of four patients with polyneuropathy and secondary hypothyroidism are reported. Neurophysiological studies revealed signs of muscle denervation and reduction of conduction velocity in all the patients. Sural nerve biopsies showed axonal degeneration in all cases but one. All the patients were treated with replacement therapy and clinical symptomatology and neurophysiological parameters improved in all patients.

In the literature two types of abnormality of the peripheral nervous system in hypothyroidism have been described. The first is a mononeuropathy, due to mucinous deposits which cause nerve damage through a compression mechanism; ${ }^{1}$ the second is a sensorimotor polyneuropathy. Morphologically, some studies have shown a primary involvement of myelin, ${ }^{23}$ while more recent studies have shown primary axonal damage. ${ }^{45}$ The present report deals with four patients with sensorimotor polyneuropathy associated with hypothyroidism. These patients were treated by substitution therapy and followed clinically and electrophysiologically until achievement of the euthyroid state.

\section{Case reports}

Patient 1 G.B., a 33 year old woman presented with periorbital oedema, weakness first of the lower limbs and then of the upper limbs, distal cramps and paraesthesia. Subsequently, there was dysphonia, dysarthria and reduction of libido. Two months after onset the patient was hospitalized. On general examination, she showed only periorbital oedema and dry skin. Neurological examination showed slight dysarthria and dysphonia, mild hypertrophy of the calf triceps and bilateral reduction of knee and ankle reflexes. Muscle strength and sensory examination were nor-

Address for reprint requests: Raffaello Nemni, M.D., Department of Neurology, S. Raffaele Hospital, Via Olgettina 6020132 Milan, Italy

Received 2 December 1986 and in revised form 23 March 1987. Accepted 2 April 1987 mal. Laboratory data were normal except for a moderate normochromic and normocytic anaemia. CK and LDH were normal. T3 was $0.2 \mathrm{ng} / \mathrm{ml}$ (normal $0.6-2.0 \mathrm{ng} / \mathrm{ml}$ ), T4 was $13 \mathrm{ng} / \mathrm{ml}$ (normal $65-130 \mathrm{ng} / \mathrm{ml}$ ); TSH was $63 \cdot 3 \mathrm{IU} / \mathrm{ml}$ (normal 2-7 IU $/ \mathrm{ml}$ ). Antimicrosomal, antismooth muscle and antigastric wall antibodies were found. Ultrasound and scintiscanning showed a small thyroid gland with poor uptake. The diagnosis was Hashimoto's thyroiditis.

Patient 2 E.B., a 58 year old woman, was admitted because of a 5 month history of weakness and paraesthesia in upper and lower limbs. There was no significant past history, except for recent constipation. The general examination showed a dry and cold skin, alopecia on the outer third of the eybrows and a goitre. Neurological examination disclosed dysarthria, slight weakness of all four limbs, especially in the distal muscles of the legs. Knee and ankle reflexes were depressed. She had widebased standing and her gait was slightly ataxic. There was no sensory impairment. Laboratory data were normal except for T3 $0.8 \mathrm{ng} / \mathrm{ml}$; T4 $12 \mathrm{ng} / \mathrm{ml}$ and TSH $35.1 \mathrm{IU} / \mathrm{ml}$. Organ specific autoantibodies were absent. Thyroid scintiscanning showed a reduced uptake of radioisotope Tc 99 .

Patient 3 E.M., a 58 year old man, was seen 2 years after the onset of weakness in the proximal muscles of the legs, distal cramps caused by exposure to cold and fasciculation. A subacute thyroiditis had been diagnosed 4 years before. Neurological examination revealed mild deterioration of higher cerebral functions, dysarthria and scanning speech. The only abnormalities in the upper limbs were reduced tendon reflexes. In the legs, both bulk and strength were reduced, with slight atrophy of both quadriceps muscles and fasciculation. Knee and ankle jerks were absent. Touch and vibration sensations were slightly diminished in all four limbs. Laboratory investigations showed normochromic 
and normocytic anaemia; cholesterol level was close to the upper limit of normal; endocrinological assays could not detect T3 and T4. TSH was $64 \mathrm{IU} / \mathrm{ml}$. There were only antigastric wall antibodies. In the thyroid scintiscan iodine uptake was markedly reduced. The ECG showed a sinus bradycardia with P-wave, due to early left auricle dilatation. Patient 4 S.B., a 59 year old woman, had been treated 10 years previously for Graves disease by radiotherapy. She was admitted because of a 3 year history of paraesthesia and cramps in the lower limbs and later in the upper limbs. Muscle strength in her legs was very weak. There was a past history of depression and hallucinations after radiotherapy, treated with haloperidol. Examination showed that muscle bulk and strength were reduced more in the lower than in the upper limbs. Neither knee nor ankle jerks could be elicited. There were touch sense anaesthesia and vibration sense impairment distally in the legs; joint-position sense was decreased in her toes and calves. The patient was unable to walk without support. The laboratory data revealed a macrocytic anaemia, with a negative Shilling's test and normal folate and B12 levels. T3 was $0.8 \mathrm{ng} / \mathrm{ml}$. T4 was $22 \mathrm{ng} / \mathrm{ml}$; TSH was $18 \mathrm{IU} / \mathrm{ml}$. There were no organ specific antibodies.

\section{Methods}

Neurophysiological studies were performed in all the patients. Ulnar and deep peroneal motor conduction velocities and median and sural nerve sensory conduction velocities were measured. Stimulation and recordings were done with surface electrodes. The examination was performed in a warm room $\left(24^{\circ} \mathrm{C}\right)$. Cutaneous temperature was monitored at $36^{\circ} \mathrm{C}$ by a thermostat driving a heating lamp.

A right femoral quadriceps biopsy was taken from Patient 2 and the specimen of the vastus lateralis muscle was divided into two portions. The first segment was fixed in $10 \%$ formalin, embedded in paraffin, sectioned and stained with H\&E. The second one was frozen, sectioned and stained with Gomori trichrome, ATPase ( $\mathrm{pH} \mathrm{10.4)}$ and NADH-TR, for the histochemical study by light microscopy.

Sural nerves were taken in toto from the ankle of each patient and the specimens divided into two portions; the first was fixed in $10 \%$ formalin, washed, dehydrated, infiltrated, embedded in paraffin, sectioned and stained with $H \& E$, PAS, alcian blue, and congo red. The second was fixed in buffered $2 \%$ glutaraldehyde for 2 hours, postfixed in buffered 1\% osmium; dehydrated and embedded in Epon 812. Semithin sections were stained with toluidine blue and examined under light microscope. Thin sections were doubly stained in $5 \%$ uranyl acetate and in $0.4 \%$ lead citrate for electron microscopy. Quantitative histometry of myelinated fibres were obtained from micrographs of three fascicles for each patient, at a magnification of $\times 1000$.

After 3 or 6 months, all patients had a clinical and electrophysiological check up to assess the effects of hormone treatment on peripheral nerve involvement.

\section{Results}

Electrophysiological study In all patients, the electromyograms disclosed a pattern of neurogenic damage and the electroneurographic examination demonstrated increased distal latencies of nerve action potentials and moderate slowing of conduction velocities. The electroneurographic data were summarised in table 1 .

MORPHOLOGICAL EXAMINATION OF THE SURAL NER VE

Light microscopy None of the patients had any inflammatory infiltration, or epineurial, perineurial or endoneurial abnormalities in paraffin sections. There was no evidence of abnormal deposits after congo red, PAS or alcian blue staining.

Resin embedded sections of Patient 1 showed normal morphology. The quantitative histometric analysis showed a normal endoneurial area. The total number of myelinated fibres was 7056 (normal $5200-9500$ ) and their density was $7840 / \mathrm{mm}^{2}$ (normal $5400-8600 / \mathrm{mm}^{2}$ ). The distribution of diameters of myelinated fibres was normal. The sural nerve of

Table 1 Nerve conduction in hypothyroid patients

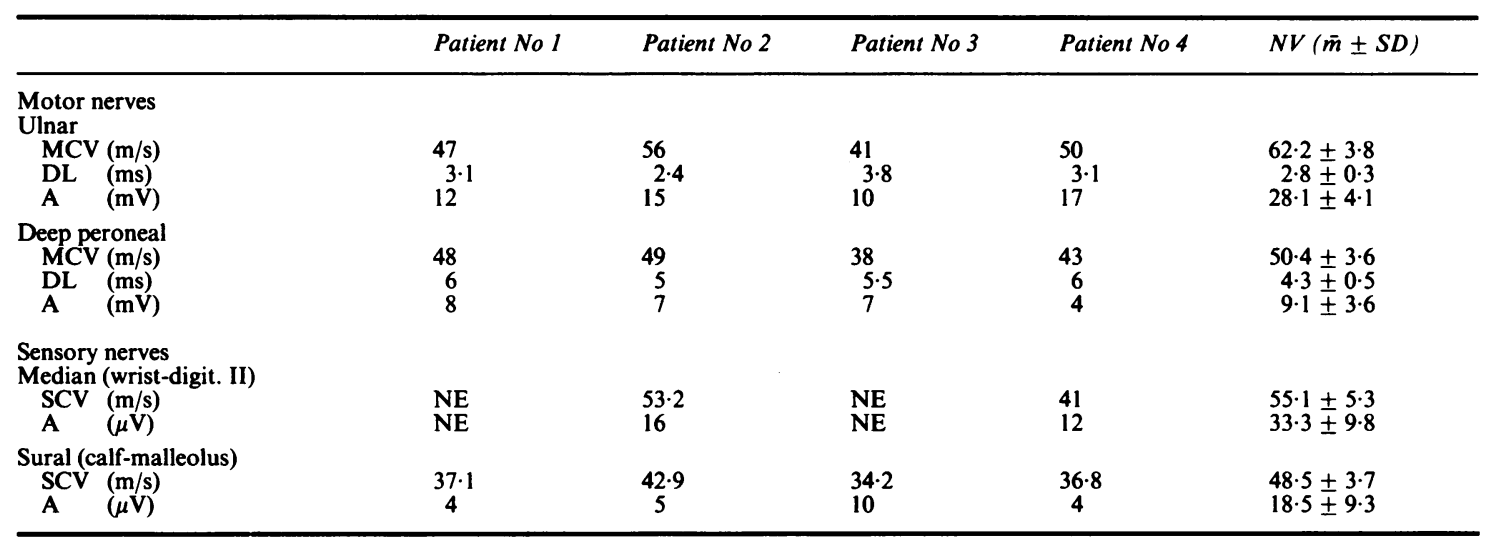




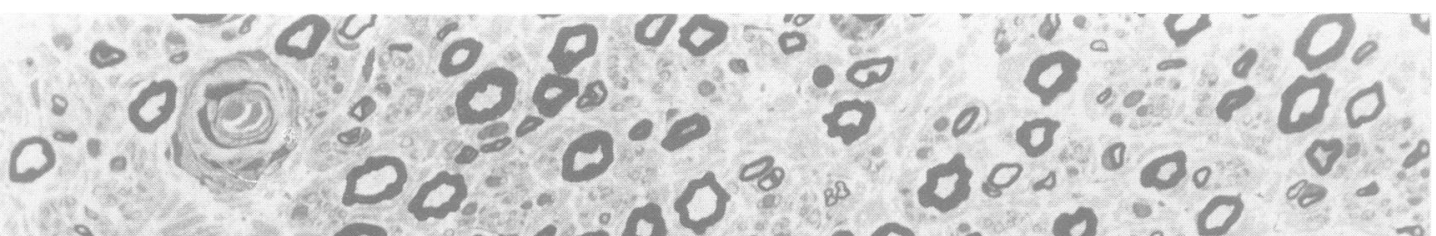

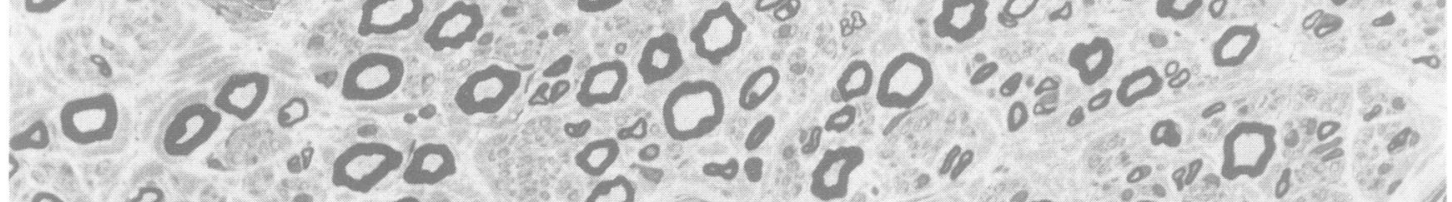

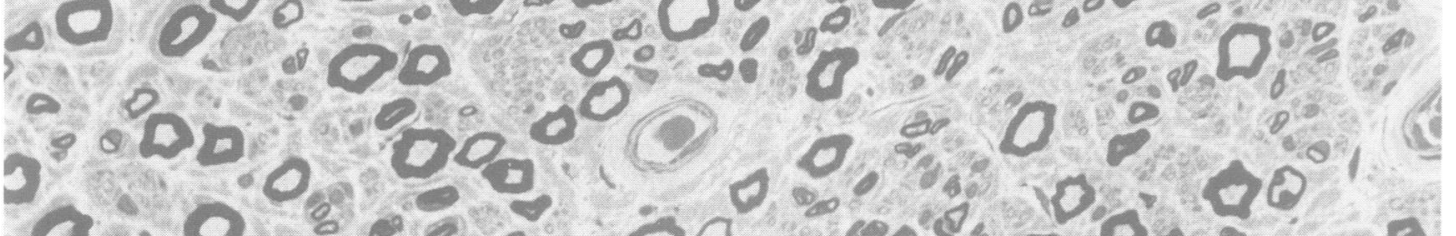

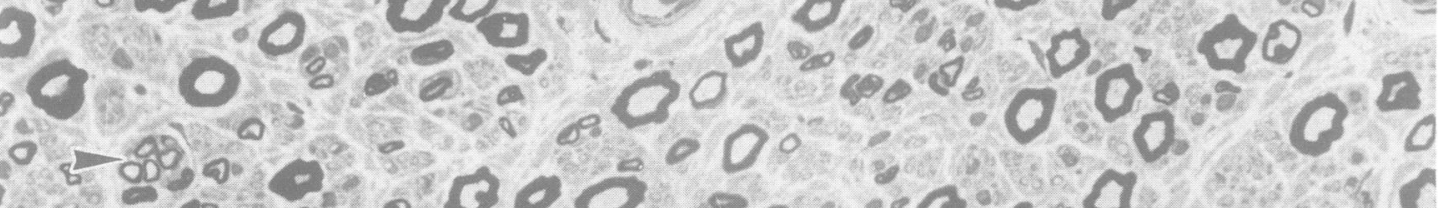
$3: 9.80000=0.0000$ - $0000^{\circ}$ o m. OS.000.

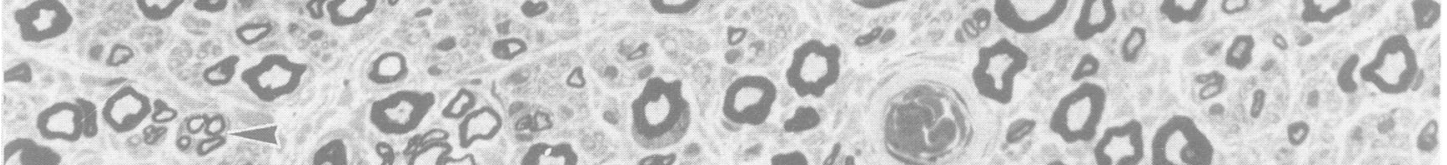

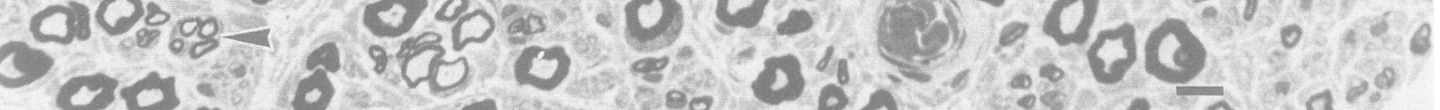

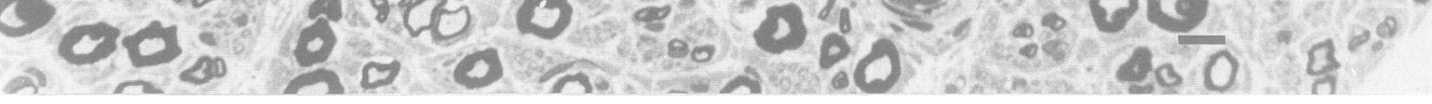

Fig 1 Transverse section of sural nerve, patient 3. Moderate endoneurial fibrosis, with non-selective reduction of number of myelinated fibres. Some clusters (D) are present. Epon-embedded semithin section, toluidine blue; Bar = $10 \mu m$.

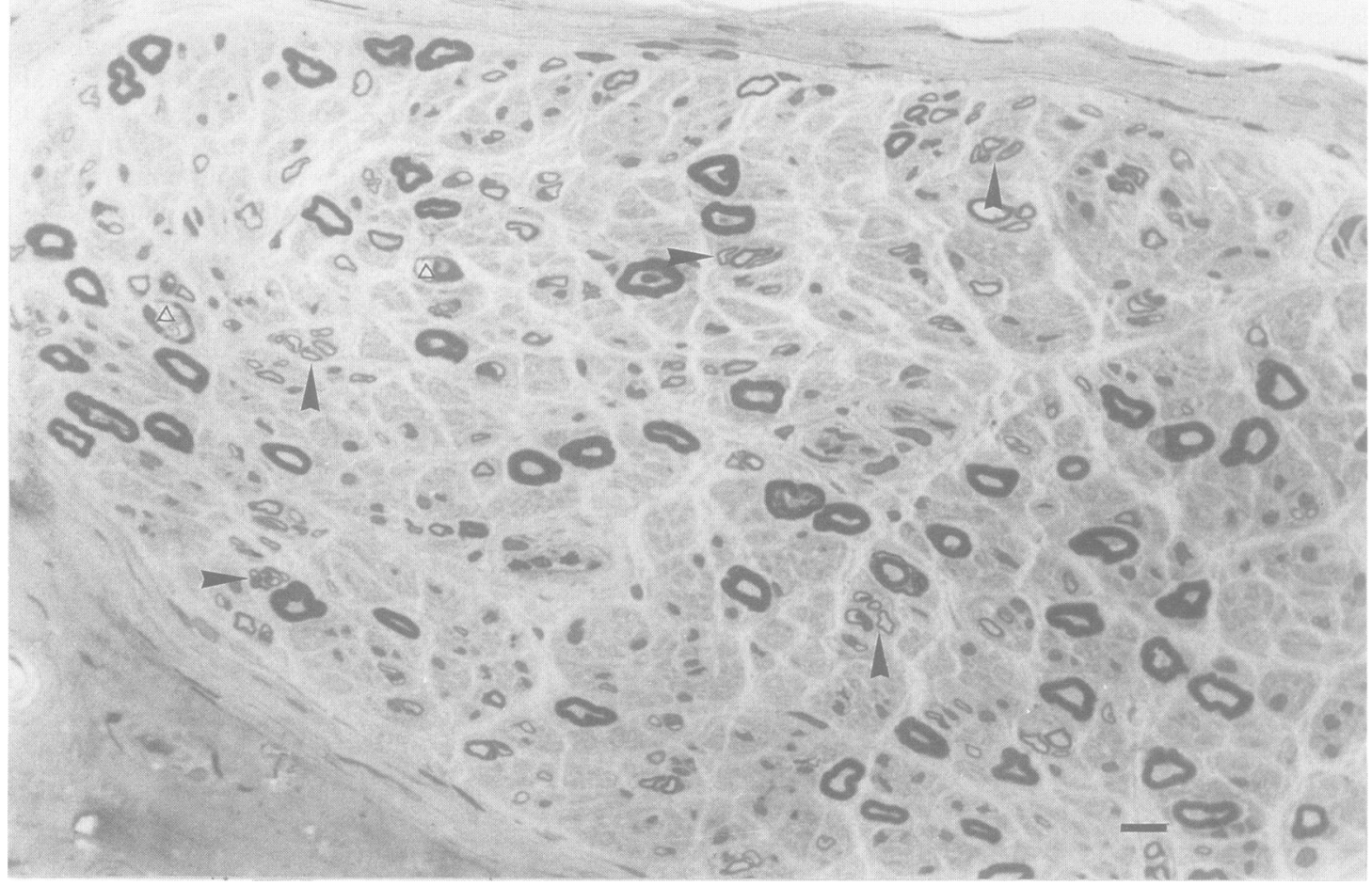

Fig 2 Transverse section of sural nerve, patient 4. Marked loss of myelinated fibres and remarkable endoneurial fibrosis. A few degenerating fibres $(\triangle)$ and some clusters of myelinated fibres $(\checkmark)$ are present. Epon-embedded semithin section, toluidine blue; Bar $=10 \mu \mathrm{m}$. 


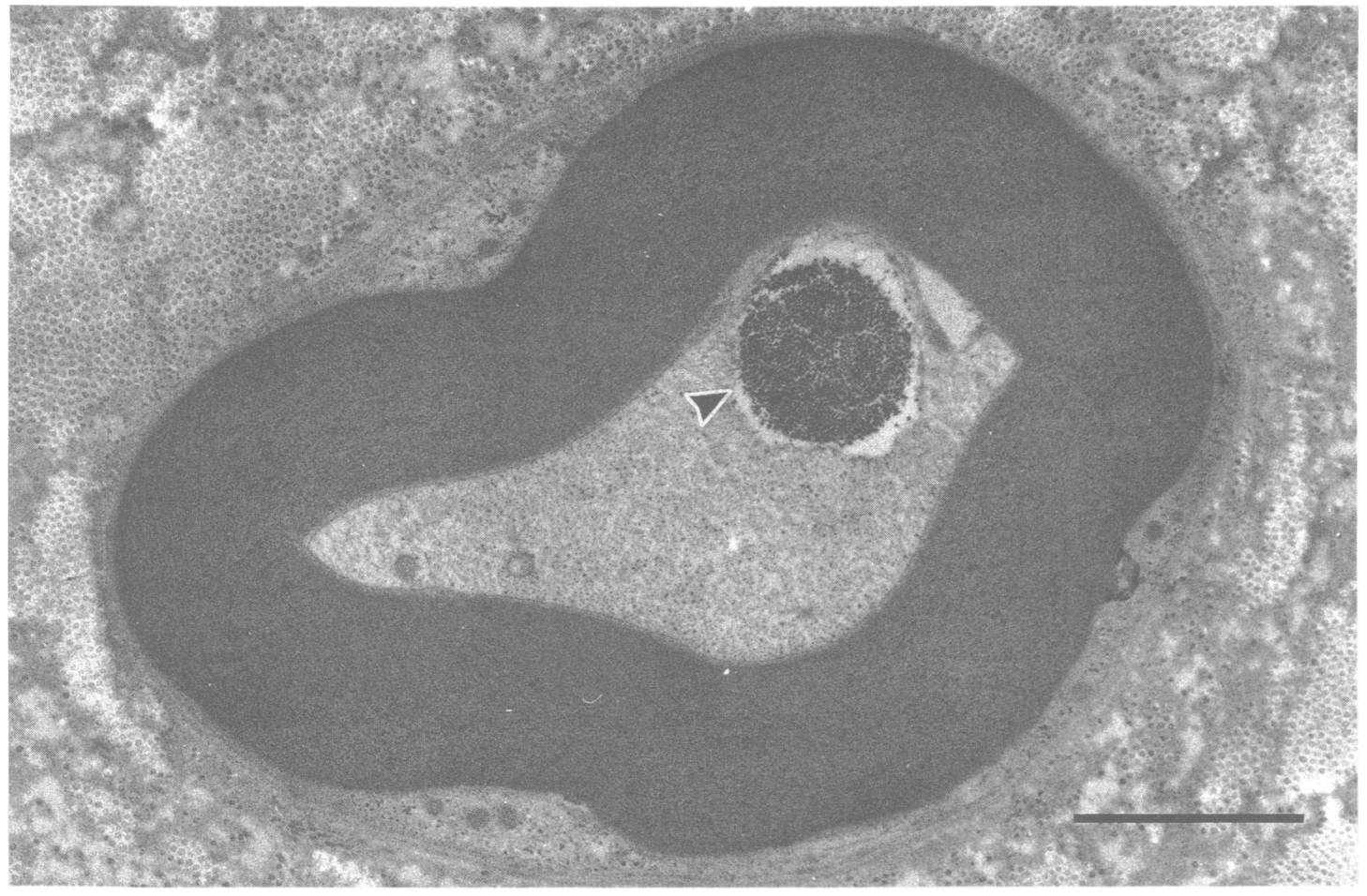

Fig 3 Sural nerve biopsy specimen, patient 4. A myelinated fibre with vacuolated axoplasm and glycogen aggregates ( ). Bar $=1 \mu \mathrm{m}$.

Patient 2 showed a few degenerating fibres. Quantitative histological measurements demonstrated a normal endoneurial area, an increase in total number of myelinated fibres (9662) and in their density $\left(11,785 / \mathrm{mm}^{2}\right)$. Small diameter myelinated fibres were responsible for this increase, demonstrated by the percentage of fibres less than $8 \mu \mathrm{m}(86.7 \%$; normal $66 \cdot 4-77 \cdot 2 \%$ ). The sural nerve of Patient 3 revealed endoneurial fibrosis with moderate loss of myelinated fibres of all diameters. There were some clusters of small myelinated fibres and large diameter axons with granulated axoplasm (fig 1). By quantitative histometry the endoneurial area was normal. The total number of myelinated fibres was 4208 and their density was 5246. Both large and small myelinated fibres decreased similarly.

The transverse sural nerve sections of Patient 4 exhibited a marked reduction of myelinated fibres and remarkable endoneurial fibrosis. Many residual fibres had abnormal changes in the axoplasm, with granulated appearance and increased density, while other fibres were acutely degenerating (fig 2 ). In addition, numerous Renaut bodies were found. Some fibres were remyelinated. In this case, histometrical findings demonstrated a normal endoneurial area. The total number and density of myelinated fibres were uniformly and markedly reduced to 2653 and 3765 respectively.

Electron microscopy This confirmed the absence of pathological alterations in Patient 1 and the presence of only a few degenerating fibres in Patient 2. Patients 3 and 4 had exclusively axonal damage, with abnormal axonal organelles, disintegration of microtubules and neurofilaments and vacuolisation of axoplasm with glycogen aggregates (fig 3). These alterations were found in axons of all diameters. Images of Bungner's bands were frequent. Empty Schwann cell processes suggesting pathological involvement of unmyelinated fibres, were numerous (fig 4). The nuclei and cytoplasm of Schwann cells were normal in appearance. There were none of the mitochondrial abnormalities observed by other authors. ${ }^{2}$

\section{Morphological muscle study}

With histochemical stains, many target fibres and numerous angulated fibres were superdark with 


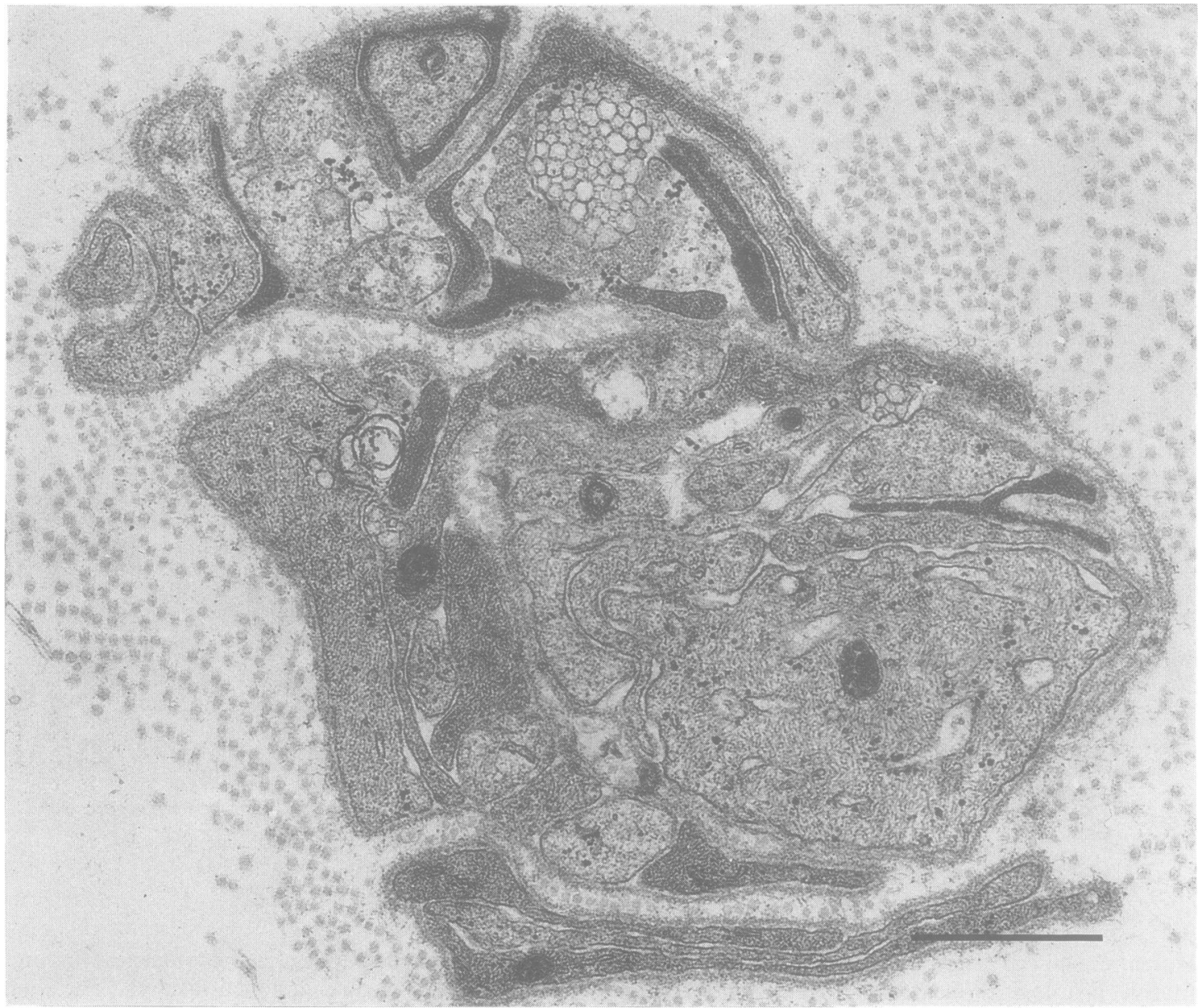

Fig 4 Sural nerve biopsy specimen, patient 3. Empty Schwann cell processes, a sign of pathological involvement of unmyelinated fibres. Bar $=1 \mu \mathrm{m}$.

NADH-TR, indicating acute neurogenic muscular atrophy (fig 5).

At follow up after 3 months of hormone treatment, neurological examination of Patients 1 and 2 were completely negative. After 6 months, the examination of Patient 3 revealed only decreased tendon reflexes in the legs. After 6 months of substitution therapy, there were no important changes in the clinical picture of Patient 4 . She had slight recovery from the muscle weakness in the lower limbs and the depression and hallucinations had disappeared. After 3 months of therapy, all the neurophysiological parameters of Patients 1 and 2 were normal. After 6 months of therapy Patients 3 and 4 had only some improvements on ENG data. (table 2).

\section{Discussion}

Median nerve entrapment at the wrist caused by the deposition of mucinous material in the tissue surrounding the nerve is one of the most frequent causes of peripheral nerve damage in hypothyroidism. A similar mechanism of nerve damage has been postulated for peripheral neuropathy, although that is not yet supported by adequate morphological evidence.

Dyck and Lambert ${ }^{2}$ studied two cases morphologically and neurophysiologically and suggested that metabolic alterations caused by endocrine disorders are responsible for the peripheral neuropathy. They suggested that these metabolic alterations affect essentially the Schwann cell inducing a segmental demye- 


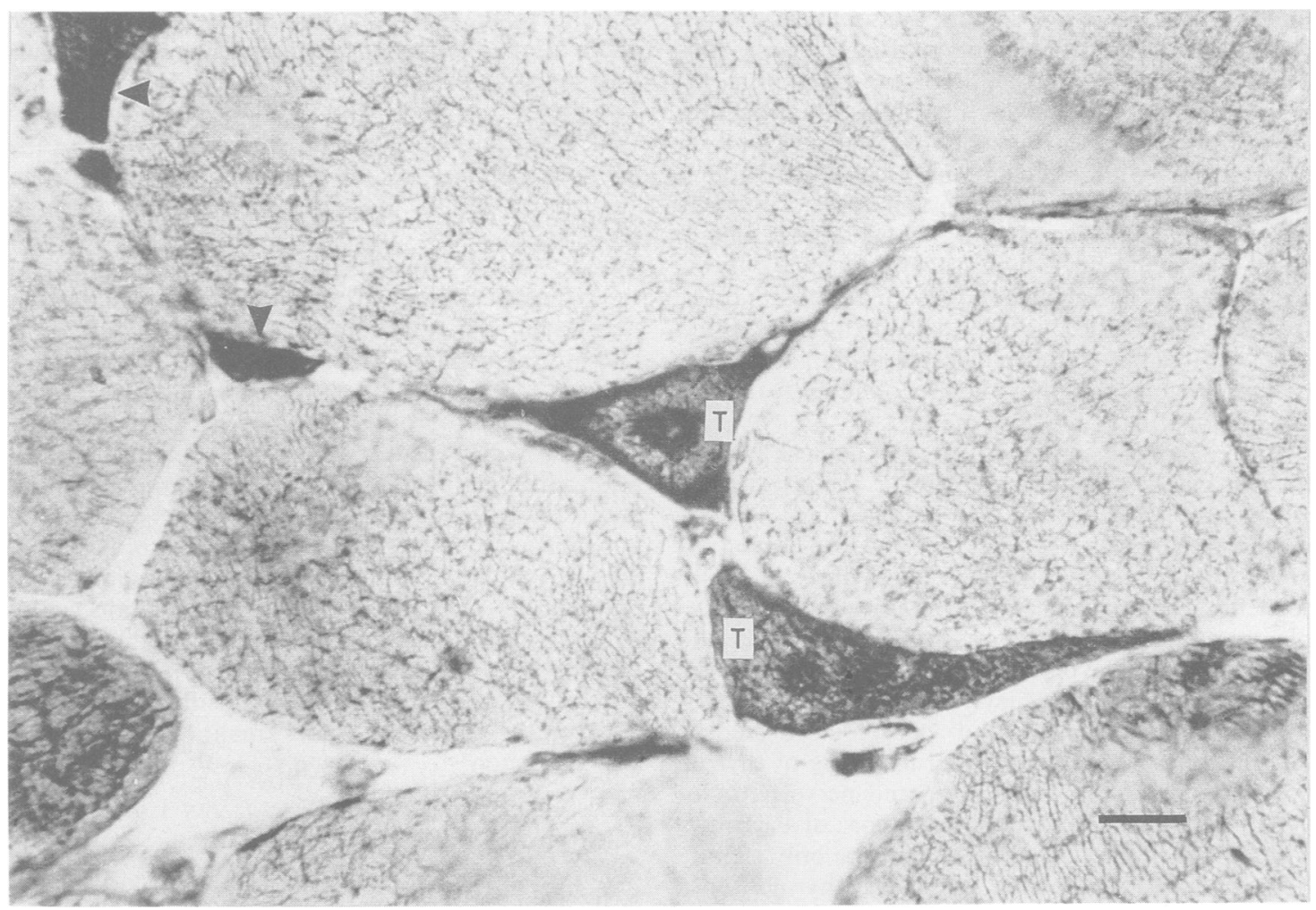

Fig 5 Brachial biceps muscle biopsy, patient 2. Target fibres (T) and small angulated fibres ( ) indicate acute neurogenic alterations. NADH-TR; Bar $=10 \mu \mathrm{m}$.

Table 2 Nerve conduction studies in euthyroid patients: follow-up after 3-6 month therapy

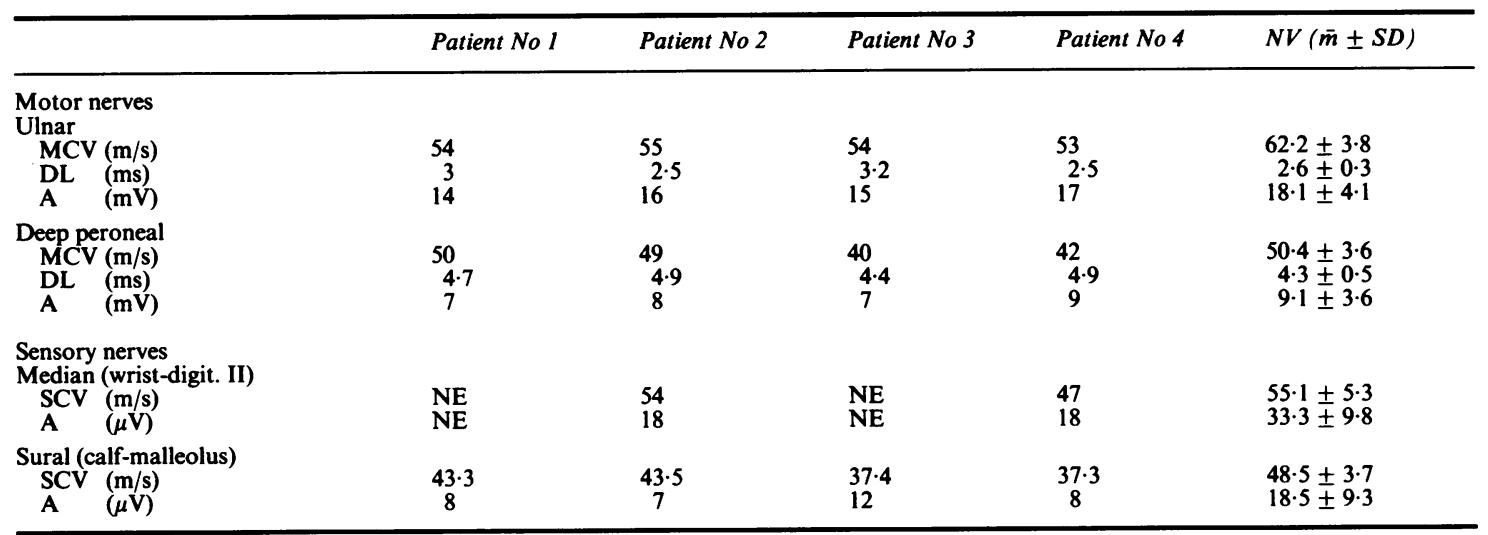


lination. Shirabe et $a l^{3}$ came to the same conclusion. Recent observations have not confirmed the previous ones. Some investigators ${ }^{45}$ found morphological evidence of primary axonal degeneration. In teased fibres they demonstrated arrays of myelinated ovoids in most fibres; with electron microscopy they found shrinkage of axons, disintegration of neurotubules and neurofilaments and active axonal break down. Segmental demyelination was also present, but it was considered to be secondary to axonal degeneration. On the other hand, Dyck and Lambert also reported evidence for axis cylinder disease, although, in their opinion, demyelination is the predominant feature of the neuropath.y.

Both neurophysiological and pathological findings for our cases are consistent with a primary axonal degeneration. Clinically our patients had sensorimotor polyneuropathy, with a distoproximal progression that first involved the lower limbs and subsequently the upper ones. The severity of the clinical picture of the neuropathy was more strictly related to the duration of the disease than to the severity of the thyroid hormone deficiency. This was also confirmed by electrophysiological and pathological studies. Patient 3, with undetectable serum levels of thyroid hormone, had symptomatology and nerve pathological alterations less serious than those of Patient 4, who, although her hormone value were only slightly below normal limits, had been hypothyroid for a longer time.

In all the patients, neurophysiological examination demonstrated a reduction in MAPs and SAPs amplitude and mild slowing of the sensory and motor conduction velocities, consistent with the presence of axonal neuropathy.

Though Patient 1 had neurophysiological abnormalities, she was normal according to the morphological study. Patient 2 had predominant signs of axonal degeneration in the sural nerve biopsy and acute abnormalities in the muscle biopsy. In the sural nerve of Patients 3 and 4, we saw striking axonal alterations.

Comparing the electrophysiological and morphological data, there was early occurrence of functional rather than of structural abnormalities. In all our patients the distal nerve fibres were initially affected. In Patient 2 especially the nerve endings in the lower limbs were preferentially affected.

In hypothyroidism, the energy deficit is due to the decrease in oxidation and the glycogen deposits are due to decrease in degradation. Under physiological conditions, the thyroid hormone is responsible for the stimulation of mitochondrial respiratory activity, thus helping production of energy, in the form of ATP, during aerobiosis. Thyroid hormone seems to increase ATPase activity and, consequently, the activity of the ATP-dependent $\mathrm{Na}^{+} / \mathrm{K}^{+}$pump. The increase in ATPase activity would be associated with an increase of ATP transport through the mitochondrial membranes. ${ }^{6}$ In hypothyroidism, the ATP deficiency and the reduced activity of the ATPase enzyme induces a decrease in $\mathrm{Na}^{+} / \mathrm{K}^{+}$pump activity, with consequent alterations of pump-dependent axonal transport. A reduced axonal velocity of slow component a in sciatic nerves of hypothyroid rats has been recently demonstrated which, it is suggested, leads to axonal degeneration and peripheral neuropathy. ${ }^{7}$ Our data are consistent with this pathogenetic mechanism since the polyneuropathy we found may be interpreted as a dying-back neuropathy, both clinically and morphologically. The metabolic alterations induced by hypothyroidism may initially damage function in the nerve (Patient 1) and only later induce structural alterations. In all cases but one, substitution therapy reversed the biochemical abnormalities and restored nerve function.

This work was supported by grants from Istituto Scientifico S. Raffaele, Milan.

\section{References}

1 Murray ICP, Simpson JA. Acroparaesthesia in myxoedema: a clinical and electromyographic study. Lancet 1958;1:1360-3.

2 Dyck PJ, Lambert EH. Polyneuropathy associated with hypothyroidism. J Neuropathol Exp Neurol 1970;24: 631-57.

3 Shirabe T, Tawara S, Terao A, Araki S. Myxoedematous polyneuropathy: a light and electron microscopic study of the peripheral nerve and muscle. J Neurol Neurosurg Psychiatry 1975;38:241-7.

4 Meier C, Bischoff A. Polyneuropathy in hypothyroidism. Clinical and nerve biopsy study of four cases. $J$ Neurol 1977;213:103-14.

5 Pollard JD, McLeod JG, Honnibal TGA, Verheijden MA. Hypothyroid polyneuropathy. J Neurol Sci 1982;53:461-71.

6 Ingar SH, Woeber KA. The thyroid gland. In: William RH ed. Textbook of Endocrinology. Philadelphia WB Saunders. 1968:105-286.

7 Sidenius P, Laurberg P, Nagel P, Larsen JR, Boye N. Reduced axonal transport velocity of slow component a in sciatic nerves of hypothyroid rats. PNAA Meeting, November 4-8, 1986, SC: 46 (Abstract). 